\title{
Construction and application of temperature and humidity precision control model based on differentiation theory
}

\author{
Shengli Ye ${ }^{1}$, Yunfei Xia ${ }^{2 *}$, Zhengguo Hong ${ }^{3}$ and Hangping $\mathrm{Jin}^{4}$ \\ ${ }^{1234}$ China Tobacco Zhejiang Industrial Co., Ltd, Hangzhou, China
}

\begin{abstract}
Through the construction and application of the temperature and humidity precision control model of the differentiation theory, and on the basis of customer needs, the temperature and humidity precision control model based on the differentiation theory is proposed. The core concept is to implement differential control in time and zone, and use zone control, Card edge control, dual-mode control and other control methods to achieve stable, reliable and precise control of the temperature and humidity of the production environment, so as to reduce the energy consumption of the air conditioning system.
\end{abstract}

\section{Foreword}

With the rapid development of China's economy, China's energy resources are becoming increasingly tight, and energy conservation and emission reduction have risen to the national strategic level. Among them, industrial enterprises have always been my country's major energy consumers. According to the "China Statistical Yearbook" data, industrial energy consumption has accounted for more than $70 \%$ of the country's energy consumption in recent years, and industrial enterprises have always been important targets for energy conservation and emission reduction. Similarly, with the development of the industry, energy consumption in the tobacco industry also brings high energy consumption in production. In particular, the annual average energy consumption of cigarette manufacturing plants often reaches the level of local key energy-consuming units[1]. The industry's energy-saving and emission-reduction potential and space are still great, and the energy consumption of the air-conditioning system is the main energy-consuming equipment of each tobacco factory, which generally accounts for $40 \%$ to $60 \%$ of the energy consumption of the whole factory. The airconditioning system is the focus of energy-saving and emission-reduction work for cigarette manufacturing factories[2].

Therefore, this article uses the precision temperature and humidity control model of the differential theory to implement precise energy supply for the process air conditioning of the cigarette manufacturing plant.

\section{Application of zone control in precise control of temperature and humidity}

In order to achieve precise control of temperature and humidity, to solve the two problems of temperature control, temperature and humidity and reduce energy consumption, the project team developed a lean control method for variable temperature and humidity. The core concept is: different areas, different control, including the following three steps:

\subsection{Accurate identification of differentiated areas}

By identifying the needs of external customers, such as the silk workshop, rolling package, and production technology department, the project team divided the areas that need temperature and humidity control into two categories: the first type of area, that is, the key area, with high control accuracy as the goal. The second-class area, that is, the general area, can save energy and reduce consumption as much as possible while ensuring stable temperature and humidity control. For key areas, the project team adopts the central point control method, and for general areas, adopts the interval control method first used in the industry[3].

\subsection{Reasonable establishment of quantitative indicators}

A more reasonable setting value is needed for the air conditioning control target, that is, interval setting. This setting requires comprehensive tests based on factors such as the heat generation of equipment in different production areas, the degree of fluctuations in the external environment, and air-conditioning conditions. Long-term experience and data accumulation can define the range of quantitative results for air-conditioning control targets in different production areas.

\subsection{Differentiated control plan}

The principle of the central point control method is shown

"Corresponding author's e-mail: xiayunfei@zjtobacco.com 
in Figure 1. For areas that require temperature and humidity control, the factory has formulated corresponding specification lines. For example, the factory requires that the upper limit of the temperature of a room is 27 degrees Celsius and the lower limit is 21 degrees Celsius. The center point control method is to take the center value of the upper and lower limits as the control target value, which is 24 degrees Celsius. Once the on-site temperature sensor detects a deviation from 24 degrees Celsius, it will drive the corresponding valve to adjust to keep it close to 24 degrees Celsius. The control accuracy of this control method is relatively high, but it consumes more energy. In the figure, the blinking yellow dots are in line with our temperature and humidity control requirements, but the central point control method needs

Flow chart of

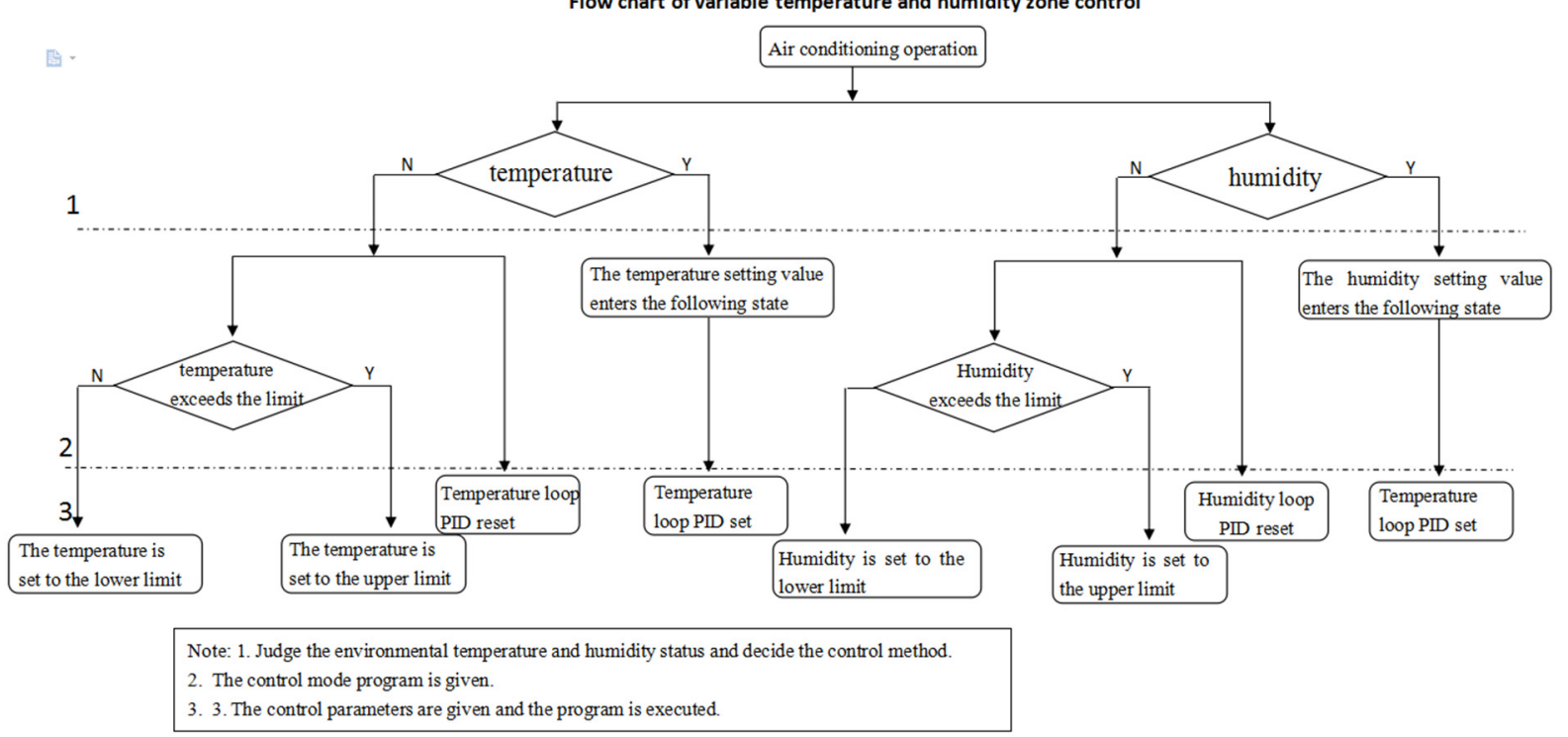

Figure 2 Process of temperature and humidity zone control

\subsection{Application effect}

After the implementation of the project, approximately 7.2 million energy costs can be saved per year. At the same time, the original result-returning energy consumption control mode is transformed into a process control method, making energy consumption management more refined.

\section{Application of multi-mode control in precise control of temperature and humidity in elevated warehouses}

In order to reduce the energy consumption of air- to be adjusted, resulting in Larger energy consumption.

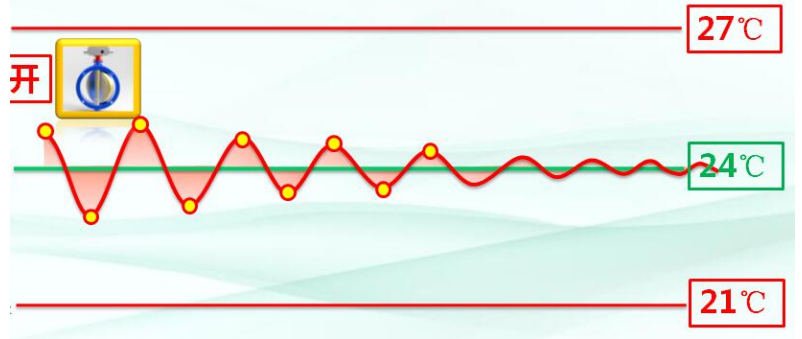

Figure 1 Principle of temperature and humidity center point control method

The process of temperature and humidity zone control is shown in Figure 2.

temperature and humidity zone control 


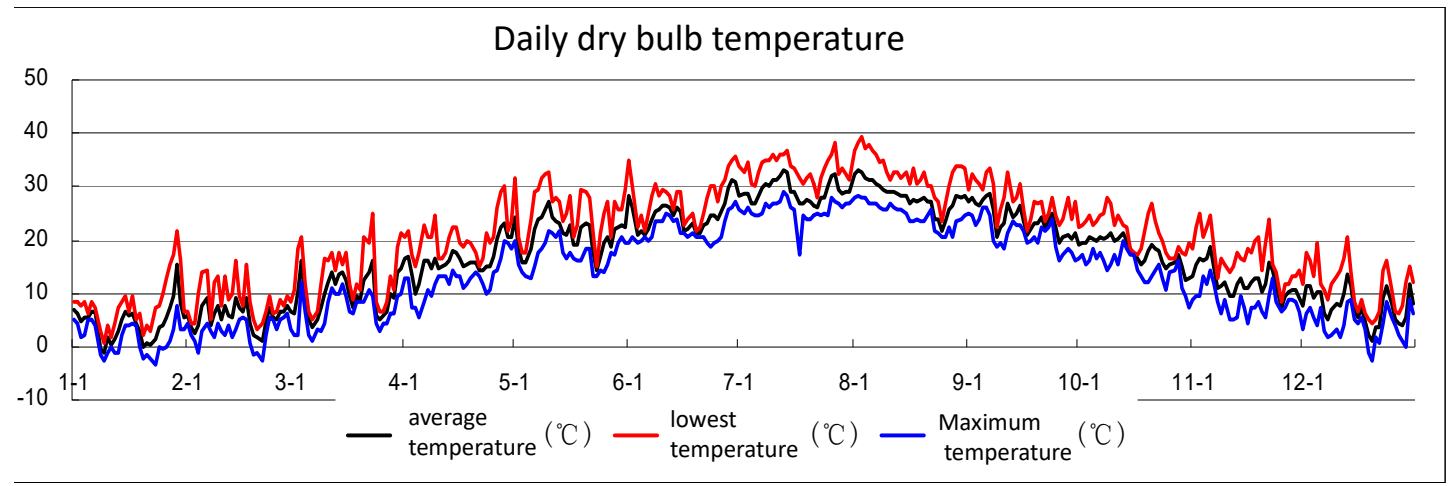

Figure 3 Typical annual temperature distribution in Hangzhou

The HDY-SMAD HVAC load calculation and analysis and heating load and design air supply parameters of the software V3.5 is used to simulate and calculate the cooling finished tobacco high-bay warehouse.

Table 1 Simulation results of cooling and heating load

\begin{tabular}{|c|c|c|c|}
\hline \multirow{2}{*}{$\begin{array}{l}\text { Summer } \\
\text { conditions }\end{array}$} & Roof cooling load/W & $\begin{array}{c}\text { External wall cooling } \\
\text { load/W }\end{array}$ & Indoor heat disturbance/W \\
\hline & 24804.7 & 10549.7 & 4650.7 \\
\hline \multirow{2}{*}{ Winter conditions } & Roof heat load/W & External wall heat load/W & Indoor heat disturbance/W \\
\hline & 30565 & 19592 & 4650.7 \\
\hline \multicolumn{4}{|c|}{ Design air supply parameters } \\
\hline \multicolumn{2}{|c|}{ Calculation parameters } & rature $/{ }^{\circ} \mathrm{C}$ & Air supply $/ \mathrm{m} 3 / \mathrm{h}$ \\
\hline \multicolumn{2}{|c|}{ Summer air supply parameters } & & 100000 \\
\hline \multicolumn{2}{|c|}{ Winter air supply parameters } & & 100000 \\
\hline
\end{tabular}

According to the indoor thermal disturbance characteristics of the elevated warehouse, the HDYSMAD HVAC load calculation and analysis software V3.5 was used to simulate the cooling and heating load of the elevated warehouse, and the air-conditioning air supply model was determined. The temperature cloud diagram of the finished tobacco in the elevated warehouse is shown in the figure below.
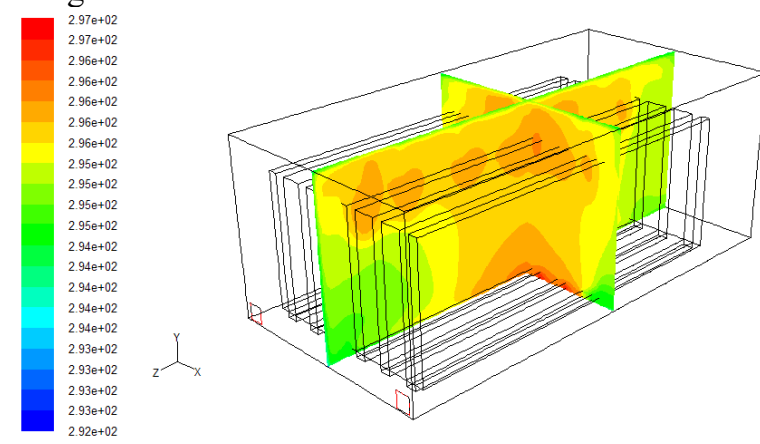

Figure 4 Temperature cloud diagram of elevated tobacco storage

In order to meet the design requirements of the elevated warehouse, based on the CFD analysis results, an air-conditioning control scheme is set. The automatic control ideas are as follows:

(1) Consider that the operation control of the air conditioning unit is divided into two modes: summer and winter. Classification basis: compare the supply air temperature (TSUPPLY) of the air-conditioning unit with the actual elevated storage temperature (TAVER). When the supply air temperature (TSUPPLY) $>$ the actual elevated storage temperature (TAVER), it is in winter mode, and the rest are in summer mode.

(2) The maximum supply air volume is used in winter mode, and the variable air volume is used in summer mode Summer variable air volume control basis: According to the simulation conclusion, the relationship between the air conditioning system load rate and the supply air volume: the ratio of the supply air volume to the design air volume $\mathrm{y} 1$ and the partial load rate $\mathrm{x}: \mathrm{y} 1=-0.1 \mathrm{x}+1.1$.

(3) Basis for controlling the air volume of upper and lower layers: separately detect the air volume of the upper and lower layers, and control the air volume of the upper and lower layers according to the relationship between the ratio of the upper and lower air supply and the load rate in different seasons. In winter mode, the air supply ratio of the upper and lower air ducts is 3.178:1; in summer mode, the relationship between the upper and lower air supply ratio $\mathrm{y} 2$ and the partial load rate $\mathrm{x}: \mathrm{y} 2=-0.042 \times 2+0.027 \mathrm{x}$ +1.794 .

Through the application of multi-mode control in the precise temperature and humidity control of elevated warehouses, the actual annual operating cost saved was approximately RMB 618,000.

\section{Application of fault-tolerant control principle in sensor self-calibration}

"Fault tolerance" means that the system is tolerant to failures. If some components fail, it will cause the control system to be abnormal or even crash the system. The faulttolerant control theory is proposed to avoid such situations. 
If the system itself can diagnose and detect the faults of equipment components, and use appropriate control strategies, it can still operate stably within a certain range, and even reach certain specific control goals, this type of system is a fault-tolerant control system.

The Network Control System (NCS) adopted by Hangzhou Tobacco Factory is a closed-loop system composed of controllers, sensors and actuators connected through a communication network. In the network control system, in view of the failure of the actuator and the sensor at the same time, while considering the influence of the delay in the network on the system performance, this project converts the uncertainty of the delay into the uncertainty of the system state equation coefficient matrix, And expand the state vector of the network control system into an augmented state vector. The distributed NCS structure based on fieldbus is shown in Figure 5.

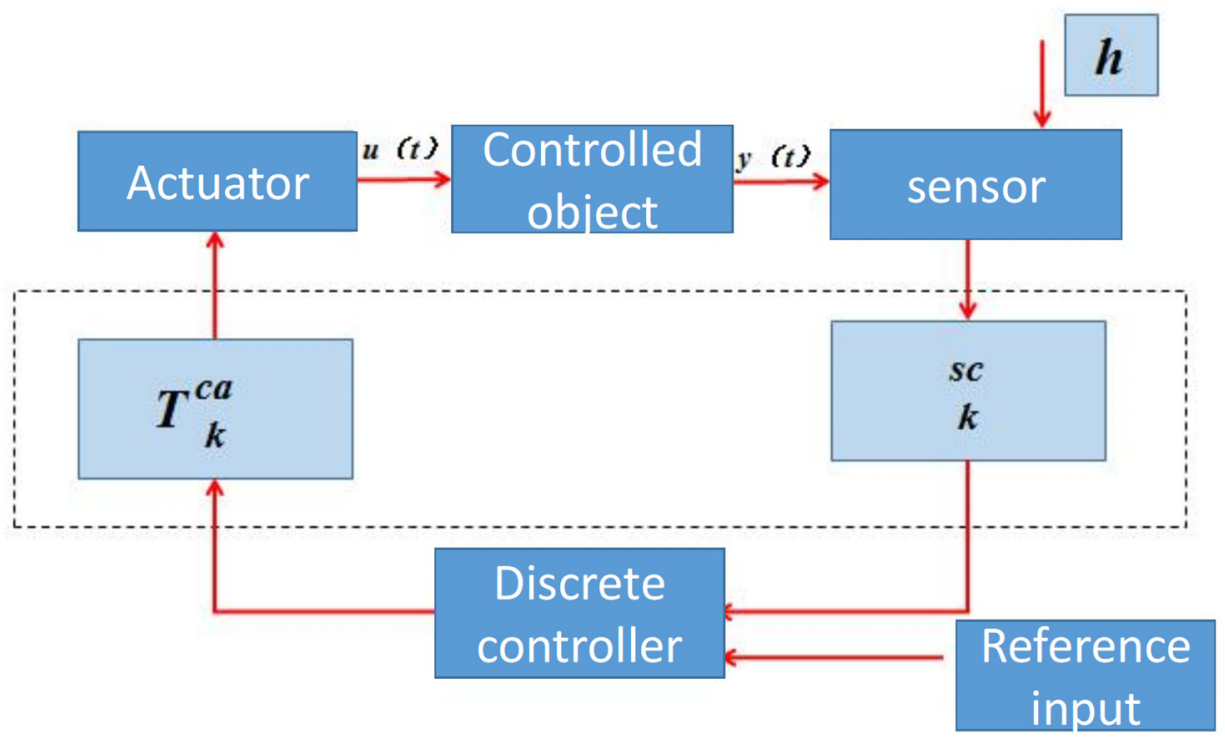

Figure 5 Distributed NCS structure based on fieldbus

Aiming at the fault situation of sensor failure, a new type of uncertain network control system fault model is established. The design method of fault-tolerant controller for networked control system based on LMI is derived by using stability theory and state feedback control. The state feedback control law obtained by this method can make the system have better robust stability in the case of sensor failure.

When the air conditioner temperature and humidity sensor meets the condition that the sensor temperature displays "-20" or the humidity displays " 0 ", the program defaults that the sensor is faulty, and the faulty sensor data is automatically removed from the calculation data collection, and the system controls according to the average value of the remaining sensors.

\section{Summary}

Through the construction and application of the temperature and humidity precise control model based on the differentiation theory, the project team proposed a precise temperature and humidity control model based on the differentiation theory on the basis of accurately identifying customer needs. The core concept is to implement differential control in time and zone. Using zone control, card edge control, dual-mode control and other control methods, the stable, reliable and precise control of temperature and humidity in the production environment is realized.

\section{References}

1. Xue Dianhua. Air conditioning [M]. Beijing: Tsinghua University Press, 1999. pp163-165.

2. United States Trane. CVH, CVHF, CVHG Watercooled CenTraVac тм cold water host installation, operation and maintenance guide $[\mathrm{M}]$. United States: Trane air conditioning company, 2008. pp163-165.

3. Tian Xudong, Liu Hua, Zhang Zhiping, etc. High temperature centrifugal chillers and their characteristics [J]. Fluid Machinery: Refrigeration and Air Conditioning, 2009,37 (10): 53-56.

4. Zhang Shuguo, Li Dong, Hu Jing. Principle and application of frequency control technology [J]. Energy Conservation Technology: 2009,27 (1): 83-86. 